\title{
CAPÍTULO 05: MÉTODOS NÃO CONVENCIONAIS DE OBTENÇÃO DE COMPOSTOS DE INTERESSE DA Stevia rebaudiana BERTONI
}

\section{CAPÍTULO 05: MÉTODOS NO CONVENCIONALES DE OBTENCIÓN DE COMPUESTOS DE INTERÉS PARA Stevia rebaudiana BERTONI}

\section{CHAPTER 05: NON-CONVENTIONAL METHODS OF OBTAINING COMPOUNDS OF INTEREST TO Stevia rebaudiana BERTONI}

\author{
Djéssica Tatiane Raspe ${ }^{1}$; Camila da Silva ${ }^{2}$; Paula Gimenez Milani ${ }^{3}$; Silvio Claudio da Costa $^{4}$
}

DOI: https://doi.org/10.31692/978-65-88970-17-1.44-62

\begin{abstract}
RESUMO
Nesta revisão, a obtenção de compostos de interesse da Stevia rebaudiana Bertoni utilizando métodos de extração não convencionais foram considerados. Os compostos obtidos dessa matriz vegetal vêm sendo amplamente reportados por exibirem benefícios terapêuticos em virtude de suas propriedades antioxidantes, antimicrobianas, antifúngicas, antitumorais e principalmente, antidiabéticas. As folhas dessa planta são utilizadas como adoçante natural e seu extrato possui constituintes com propriedades fitoterápicas, alavancando o interesse em sua investigação. Embora os métodos de extração convencionais de prensagem, maceração ou infusão sejam comumente aplicados para a obtenção destes compostos, estes processos estão associados a alguns inconvenientes, como a necessidade de longos períodos de tempo e energia, demandam o uso de solventes quentes em grandes quantidades, apresentando como consequência alguns pontos indesejáveis, como deterioração da qualidade e degradação de alguns de seus compostos. Para suprir as limitações dos métodos convencionais de extração, novas técnicas têm sido introduzidas. Essas técnicas, conhecidas como técnicas de extração não convencionais, surgem como alternativa na obtenção de compostos de interesse através de métodos como a extração assistida por ultrassom, extração assistida por micro-ondas, extração por eletrotecnologias, extração por fluido supercrítico e líquido pressurizado. Estes processos apresentam como constatações positivas o aumento no rendimento dos teores de compostos obtidos em pureza considerável, o que reduz a complexidade das etapas posteriores de purificação, oferecendo um processo mais simples, demandando de menor quantidade de energia e permitindo o uso de solventes biodegradáveis, além da diminuição dos custos envoltos ao processo, o que promove e impulsiona a "extração verde" dos compostos de interesse desta matriz vegetal.
\end{abstract}

Palavras-Chave: Adoçantes, extração verde, métodos de extração, Stevia rebaudiana Bertoni.

\section{RESUMEN}

En esta revisión se consideró la obtención de compuestos de interés para Stevia rebaudiana Bertoni mediante métodos de extracción no convencionales. Los compuestos obtenidos de esta matriz vegetal han sido ampliamente reportados por exhibir beneficios terapéuticos debido a sus propiedades antioxidantes, antimicrobianas, antifúngicas, antitumorales y, sobre todo, antidiabéticas. Las hojas de esta planta se utilizan como edulcorante natural y su extracto tiene constituyentes con propiedades fitoterápicas, potenciando el interés en su investigación. Si bien para la obtención de estos compuestos se suelen aplicar métodos convencionales de extracción de prensado, maceración o infusión, estos procesos están asociados a algunos inconvenientes, como la necesidad de largos periodos de tiempo y energía, que requieren el uso de disolventes calientes en grandes cantidades, como como consecuencia, algunos puntos indeseables, como el deterioro de la calidad y la degradación de algunos de sus

\footnotetext{
${ }^{1}$ Discente do Programa de Pós-graduação em Ciência de Alimentos (PPC), Universidade Estadual de Maringá (UEM), djessicaraspe@ hotmail.com

2 Docente do Departamento de Tecnologia (DTC), Universidade Estadual de Maringá (UEM), camiladasilva.eq@gmail.com

3 Docente do Departamento de Bioquímica (DBQ), Universidade Estadual de Maringá (UEM), pgmfernandes2@uem.br

${ }^{4}$ Docente do Departamento de Bioquímica (DBQ), Universidade Estadual de Maringá (UEM), sccosta@uem.br
} 
compuestos. Para superar las limitaciones de los métodos de extracción convencionales, se han introducido nuevas técnicas. Estas técnicas, conocidas como técnicas de extracción no convencionales, aparecen como una alternativa en la obtención de compuestos de interés mediante métodos como extracción asistida por ultrasonidos, extracción asistida por microondas, extracción por electrotecnologías, extracción por fluido supercrítico y líquido presurizado. Estos procesos tienen como hallazgos positivos el aumento en el rendimiento de los contenidos de compuestos obtenidos en una pureza considerable, lo que reduce la complejidad de las etapas posteriores de depuración, ofreciendo un proceso más sencillo, requiriendo menos energía y permitiendo el uso de disolventes biodegradables, además a la reducción de costos involucrados en el proceso, que promueve e impulsa la "extracción verde" de los compuestos de interés en esta matriz vegetal.

Palabras Clave: Edulcorantes, extracción verde, métodos de extracción, Stevia rebaudiana Bertoni.

\begin{abstract}
In this review, obtaining compounds of interest to Stevia rebaudiana Bertoni using unconventional extraction methods were considered. The compounds obtained from this plant matrix have been widely reported for exhibiting therapeutic benefits due to their antioxidant, antimicrobial, antifungal, antitumor and, above all, antidiabetic properties. The leaves of this plant are used as a natural sweetener and its extract has constituents with phytotherapic properties, leveraging the interest in its investigation. Although conventional extraction methods of pressing, maceration or infusion are commonly applied to obtain these compounds, these processes are associated with some drawbacks, such as the need for long periods of time and energy, which require the use of hot solvents in large quantities, as a consequence, some undesirable points, such as quality deterioration and degradation of some of its compounds. To overcome the limitations of conventional extraction methods, new techniques have been introduced. These techniques, known as unconventional extraction techniques, appear as an alternative in obtaining compounds of interest through methods such as extraction assisted by ultrasound, extraction assisted by microwave, extraction by electrotechnologies, extraction by supercritical fluid and pressurized liquid. These processes have as positive findings the increase in the yield of the contents of compounds obtained in considerable purity, which reduces the complexity of the later stages of purification, offering a simpler process, requiring less energy and allowing the use of biodegradable solvents, in addition to the reduction of costs involved in the process, which promotes and drives the "green extraction" of the compounds of interest in this plant matrix.
\end{abstract}

Keywords: Sweeteners, green extraction, extraction methods, Stevia rebaudiana Bertoni.

\title{
INTRODUÇÃO
}

As plantas constituem uma das fontes mais importantes de recursos terapêuticos e alimentares para uso humano. A demanda por produtos funcionais ricos em fibras, antioxidantes naturais e de baixo teor calórico tem aumentado consideravelmente na última década, devido seus efeitos benéficos à saúde (KOVACEVIC et al., 2018). Vários derivados de cereais, vegetais e inclusive subprodutos advindos de frutas, ricos em antioxidantes e fibras alimentares, têm sido propostos como aditivos funcionais para suplementação de gordura e açúcar em alimentos, vislumbrando aumento na qualidade nutricional e no valor agregado dos produtos (CARBONELL-CAPELLA et al., 2013). A stevia surge nesse contexto, não somente como um mero adoçante não calórico, mas também como um valioso item de suplementação nutricional (YU et al., 2017).

Stevia rebaudiana Bertoni pertence à família Asteraceae, é nativa do Paraguai e 
cultivada em muitos países, incluindo Brasil, Argentina, Japão, China, Estados Unidos e países europeus (DACOME et al., 2005). É importante na indústria alimentícia por suas folhas possuírem os glicosídeos de esteviol (GS) de sabor adocicado, dentre os quais está principalmente o esteviosídeo (Stv) e rebaudiosídeo A (Reb A) (CARAKOSTAS et al., 2008). Estes glicosídeos são até 450 vezes mais doces que a sacarose (WILLIAMS; BURDOCK, 2009), o que eleva seu interesse comercial. Este composto químico é termicamente estável e vêm sendo amplamente usado na indústria alimentícia de alguns países como agente edulcorante, modificador de sabor e substituto do açúcar (CARAKOSTAS et al., 2008; BRAHMACHARI et al., 2011). Não exibe toxicidade nem ação mutagênica e é uma substância de baixa caloria, não havendo efeitos adversos relatados em seu uso por seres humanos.

O valor terapêutico dos GS consiste na capacidade destes compostos estimularem a secreção de insulina no pâncreas no tratamento do diabetes e controlar outros distúrbios do metabolismo (JEPPESEN et al., 2000), além de propriedades adjuvantes no diabetes, como a redução da hiperglicemia (MILANI et al., 2017). Reporta-se que o extrato da stevia possui propriedades antivirais e produz um efeito terapêutico positivo nos tratamentos de neuralgia, anemia, lombalgia, reumatismo, eczema e dermatite (TAKAHASHI et al., 2001; CHATSUDTHIPONG; MUANPRASAT, 2009). Propriedades antibacterianas e anti-amnéicas também foram constatadas (SHARMA et al., 2010; PURI; SHARMA, 2011), e seu extrato lhe confere fonte de compostos bioativos, com propriedades antimicrobianas e antioxidantes (WOLWER-RIECK, 2012; CRIADO et al., 2014; KOUBA et al., 2015). Propriedades funcionais foram reportadas tendo-o como um composto anti-hiperglicêmico, anti-hipertensivo, anti-inflamatório, antitumoral, antidiarreico, diurético e imunomodulador (FERRAZZANO et al., 2016).

Métodos convencionais de extração de esteviosídeo das folhas da Stevia rebaudiana envolvem extração aquosa ou alcoólica, seguida de precipitação, coagulação e cristalização (PURI et al., 2011). Adicionalmente, estes processos geralmente requerem maior tempo, energia e uso de solventes orgânicos, podendo ocorrer consequências indesejáveis, como deterioração da qualidade e degradação de compostos valiosos (ŽLABUR et al., 2014; BARBA et al., 2015a). Técnicas modernas de extração, como a extração assistida por ultrassom, extração assistida por microondas, eletrotecnologias, fluido supercrítico e líquido pressurizado têm sido reportadas com destaque no processo de obtenção de produtos de interesse (KOVYLYAEVA et al., 2007; POL et al., 2007; PURI et al., 2011; CARBONELL-CAPELLA et al., 2016; GASMALLA et al., 2017; GÖRGÜÇ et al., 2019), visando evitar essas consequências na qualidade do produto e, paralelamente, desenvolver novos métodos que sejam capazes de 
melhorar o rendimento dos compostos biologicamente ativos, de modo que sejam totalmente compatíveis com o conceito de "extração verde" (CHEMAT et al., 2017).

Tendo em vista o que foi apresentado, o presente estudo visa apresentar uma abordagem sobre o aspecto atual da obtenção de compostos de interesse, como os adoçantes e bioativos, da Stevia rebaudiana Bertoni, associada à aplicação de métodos não convencionais de extração, levando em conta estudos recentes que vêm avaliando o uso destas técnicas.

\section{METODOLOGIA}

Este estudo caracteriza-se por uma revisão, pois trata-se de uma forma de pesquisa que utiliza como fonte de dados a literatura sobre determinado tema. A pesquisa de artigos com informações a respeito da "extração dos glicosídeos e compostos bioativos da stevia" analisados, consistiu-se nos bancos de dados disponíveis em serviços online, como Scopus, Scielo, Science Direct, Periódicos Capes e Google Acadêmico. As palavras-chave utilizadas foram: "Stevia rebaudiana Bertoni”, “extração verde”, "extração não convencional”, "extração alternativa", "uso de tecnologia", "glicosídeos" e "compostos bioativos", alternando o conjunto de palavras-chave de 2 a 4 por vez. Partindo disto, os critérios de inclusão e exclusão de artigos delimitou o estudo nos quesitos relevância do tema, trabalhos recentes e em inglês, bem como os que possuem disponibilidade de acesso online. Os resumos obtidos nos bancos de dados foram comparados entre si para a verificação de superposição de artigos e posteriormente, foram analisados para selecionar, ainda preliminarmente, trabalhos que atendessem aos temas propostos nesta pesquisa. Para a coleta de dados, foram selecionados resumos, os quais foram numerados sequencialmente, e uma nova etapa de busca dos artigos completos permitiu chegar à definição final dos textos que efetivamente foram analisados.

\section{RESULTADOS E DISCUSSÃO}

\section{A extração de compostos de interesse da Stevia rebaudiana Bertoni}

Em virtude da crescente conscientização dos consumidores sobre os efeitos benefícios à saúde provenientes dos alimentos, esforços têm sido desenvolvidos em prol de métodos que sejam capazes de melhorar o rendimento dos compostos biologicamente ativos e, paralelamente, compatibiliza-los ao conceito de "extração verde" (CHEMAT et al., 2017). Uma vez que estes compostos geralmente estão presentes nas plantas em baixas concentrações, métodos de extração eficientes e seletivos são necessários para a obtenção destes a partir de 
materiais vegetais. $\mathrm{O}$ primeiro passo para a obtenção de compostos bioativos a partir de matérias-primas vegetais é a extração.

A obtenção de compostos de interesse provenientes de matrizes vegetais é procedida por meio da extração sólido-líquido (ESL), cuja qual consiste em um processo de separação de um ou vários compostos presentes em uma matriz sólida e que são solúveis em um determinado solvente (MILIĆ et al., 2013). O mecanismo da ESL envolve basicamente o umedecimento da superfície do sólido com o solvente, penetração do solvente no interior do sólido, solubilização das substâncias extraíveis, transporte dos solutos do interior para a superfície do sólido e dispersão dos solutos no solvente circundante às partículas sólidas por difusão (TZIA et al., 2003; BERK, 2013).

Muitos fatores podem influenciar o rendimento da ESL, que, em métodos convencionais de extração como a prensagem, infusão, maceração, percolação, arraste por vapor e contínuo quente (Soxhlet), resumem-se na natureza química dos compostos à serem extraídos, pelo método de extração empregado, pelo solvente, pelo tamanho da partícula do material vegetal, pelo $\mathrm{pH}$ do meio extrator, pelo tempo de extração e temperatura utilizados, atrelado ao emprego de agitação, assim como pela presença de substâncias interferentes (DENG et al., 2015; PUTNIK et al., 2016; LOVRIC et al., 2017). Ademais, os métodos clássicos apresentam algumas desvantagens, como a produção de resíduos indesejáveis e a necessidade de remoção do solvente no final do processo de extração (PASSOS et al., 2010; FORMIGONI et al., 2020). Além disto, a utilização de solventes orgânicos apresenta inconvenientes relacionados à qualidade dos produtos gerados, principalmente quando correlacionados à sua pureza, bem como, a periculosidade no ambiente de produção, o impacto ambiental e custos de processamento.

Uma metodologia estudada e reportada especialmente para a stevia, que vislumbra a redução de custos e promove alternativas viáveis por meio da extração convencional, incitando facilitar e aumentar o rendimento da extração dos glicosídeos e bioativos, melhora a qualidade do adoçante obtido, além de contribuir para a redução do sabor residual amargo do esteviosídeo, envolve a utilização de pré-tratamentos químicos ou enzimáticos (PURI et al., 2012; BARBA et al., 2014; FORMIGONI et al., 2018a; FORMIGONI et al., 2018b). Porém, embora se saliente vantagens destas técnicas, o volume considerável de solvente, o custo da enzima e o destino ainda incerto para o resíduo, tornam este processo dependente de maiores avaliações. Vale ressaltar, adicionalmente, que são escassos os estudos na literatura que abordem este tema, ademais quando correlacionados aos processos de extração não convencionais. 
Visando contribuir para o cumprimento dos requisitos para o desenvolvimento de metodologias analíticas mais rápidas, eficientes, baratas, de alto rendimento e que tenham certificação GRAS (geralmente reconhecido como seguro), outras técnicas de extração vêm sendo estudadas. Essas técnicas, conhecidas como não convencionais, ditas e reportadas adicionalmente como alternativas ou modernas, são produto dos avanços dos métodos convencionais de extração ou da aplicação de novas tecnologias. A utilização da sonicação do ultrassom (ZLABUR et al., 2015; GASMALLA et al., 2017), as radiações não ionizantes do microondas (JAITAK et al., 2009; GÖRGÜÇ et al., 2019), os pulsos elétricos das eletrotecnologias (BARBA et al., 2015a; CARBONELL-CAPELLA et al., 2016), os líquidos pressurizados (YILDIZ-OZTURK et al., 2014) e fluidos supercríticos (ERKUCUK et al., 2009) têm sido reportadas com considerável destaque.

Nestes métodos, este processo é intensificado por meio do uso da tecnologia, onde cada processo apresenta sua particularidade e fatores estes que determinam sua viabilidade. Há processos que consideram a variação da temperatura, uma vez que esta variável influencia na penetração e viscosidade do solvente e solubilidade dos solutos; a aplicação de pressão, que normalmente é requerida quando se aplica alta temperatura para manter o solvente em estado líquido; o tamanho da partícula da matriz vegetal, uma vez que a redução de tamanho facilita a transferência de massa e aumenta a área de contato com o solvente; a agitação, que atua acelerando a transferência de massa; e a ruptura ou desintegração da parede celular ou dos poros das membranas celulares por influência de energia, facilitando a extração de solutos intracelulares, que geralmente são de difícil acesso por meio das técnicas convencionais (BERK, 2013; MAHNIC-KALAMIZA et al., 2014; VINATORU et al., 2017).

\section{Extração assistida por ultrassom}

A extração assistida por ultrassom (EAU) é uma tecnologia baseada em princípios sustentáveis, aplicada amplamente em processos de extração de componentes vegetais. A aplicação desta técnica permite a extração rápida de produtos naturais, que geralmente levaria horas utilizando técnicas convencionais como Soxhlet e maceração, com alta reprodutibilidade, menor consumo de solvente e facilidade de manipulação (CHEMAT et al., 2011; CHEMAT et al., 2017). Além disso, reporta-se que o equipamento de EAU possui facilidade de instalação, alta eficiência energética e requer pouca manutenção (PRADO et al., 2017).

A sonicação, efeito causado pelo ultrassom, é baseada na produção de ondas sonoras acima da faixa audível (>20 kHz), que criam bolhas de cavitação perto do tecido da amostra 
(KOUBAA et al., 2015). Quando uma grande pressão é aplicada em um líquido, as forças intermoleculares são rompidas e pequenas cavidades ou microbolhas cheias de gás são formadas. O crescimento e colapso destas microbolhas constituem no fenômeno de cavitação acústica. A implosão das bolhas de cavitação causa um impacto na superfície da matriz sólida submersa no solvente, o que ocasiona a ruptura dessa superfície. Este dano aumenta a turbulência, a penetração do solvente na matriz vegetal e causa a liberação do conteúdo intracelular (MCDONNELL; TIWARI, 2017; PRADO et al., 2017).

Uma visão geral das novas tecnologias para extrair compostos de materiais vegetais foi abortada por Heng et al. (2013), que reportam a EAU como uma técnica promissora com vários benefícios, como a aplicação em pequenos volumes de solventes à pressão atmosférica, uso de temperaturas brandas e reduzido tempo de extração, em comparação à extração convencional. É um dos sistemas de extração mais simples e baratos, podendo ser operado facilmente sob uma ampla gama de solventes (KHODDAMI et al., 2013; BARBA et al., 2015).

Neste contexto, Zlabur et al. (2015) compararam a EAU (400 W), sob diferentes diâmetros (7 e $22 \mathrm{~mm}$ ) e tempos de tratamento (2, 4, 6, 8 e $10 \mathrm{~min})$, à extração aquosa convencional $\left(100{ }^{\circ} \mathrm{C}, 24 \mathrm{~h}\right.$ ) e hidroalcoólica (etanol $70 \%, 70{ }^{\circ} \mathrm{C}, 30 \mathrm{~min}$ ), visando maximizar o rendimento em glicosídeos de esteviol, compostos fenólicos totais, flavonoides e atividade antioxidante em extratos de stevia. Desta avaliação, rendimentos na ordem de $72,7 \mathrm{mg} / \mathrm{g}^{-1}, 22,2$ $\mathrm{mg} / \mathrm{g}^{-1}, 53,9 \mathrm{mg} / \mathrm{g}^{-1}, 35,8 \mathrm{mg} / \mathrm{g}^{-1}$ e $2,1 \mathrm{mg} / \mathrm{g}^{-1}$ foram obtidos por meio da extração convencional com água, e $82,6 \mathrm{mg} / \mathrm{g}^{-1}, 24,3 \mathrm{mg} / \mathrm{g}^{-1}, 56,7 \mathrm{mg} / \mathrm{g}^{-1}, 37,9 \mathrm{mg} / \mathrm{g}^{-1}$ e $2,2 \mathrm{mg} / \mathrm{g}^{-1}$ por meio da extração convencional com etanol 70\%, em esteviosídeo, reb A, compostos fenólicos totais e atividade antioxidante respectivamente. Em paralelo, quando aplicado o ultrassom por 10 min à $22 \mathrm{~mm}$, rendimentos na ordem de $96,4 \mathrm{mg} / \mathrm{g}^{-1}, 36,9 \mathrm{mg} / \mathrm{g}^{-1}, 77,8 \mathrm{mg} / \mathrm{g}^{-1}, 62,5 \mathrm{mg} / \mathrm{g}^{-1}$ e $2,5 \mathrm{mg} / \mathrm{g}^{-1}$ foram obtidos para os mesmos compostos, comprovando a eficiência desta tecnologia por meio da maximização do processo em um tempo de tratamento significativamente inferior.

Outros autores que constataram esta mesma vantagem, realizaram uma avaliação com ênfase especial no rendimento da extração das folhas de Stevia rebaudiana Bertoni em termos de reb A. Gasmalla et al. (2017) avaliaram diferentes concentrações (10-60\%) de 3 diferentes solventes (água, etanol e álcool isopropílico) sob influência da EAU, e sob condições ótimas de extração $\left(24 \mathrm{kHz}, 18 \mathrm{~min}, 30{ }^{\circ} \mathrm{C}, 480 \mathrm{~W}\right.$ e $\left.24 \mathrm{kHz}\right)$, a recuperação de $37,10 \mathrm{~g} / 100 \mathrm{~g}$ de reb A foi possível sob um percentual de $60 \%$ de álcool isopropílico. Este rendimento obtido foi superior à técnica convencional de extração por maceração, em que rendimentos máximos de $24,21 \%$ foram obtidos (LIU et al. 2010). 
A possível implantação em escala industrial da EAU foi relatada como alternativa relevante, por se tratar de um processo rápido e sustentável ao processo convencional (VIROT et al., 2010; PINGRET et al., 2012a). Entretanto, a exposição do produto à ultrassonografia pode envolver a degradação de compostos (PINGRET et al., 2012b). Para a stevia, especificadamente, não há relatos de uma escala piloto desse tipo de tecnologia, apesar dos resultados apreciáveis de sua aplicação terem sido esboçados.

\section{Extração assistida por microondas}

Sistema de extração inovador para extrair compostos de interesse da matriz, a extração assistida por microondas (EAM) fornece alto rendimento em menor tempo, menor consumo de solvente e energia. A alta capacidade da EAM em aumentar o rendimento da extração deve-se às interações moleculares entre o componente elétrico do campo das microondas com as moléculas dipolares e as espécies iônicas presentes na mistura da extração (amostra/solvente) (MIRZADEH et al., 2020).

Essa tecnologia atua através da penetração de energia nos materiais sólidos por meio da radiação não ionizante, onde uma frequência espectral de 300-300.000 MHz gera aquecimento e dissipa-se volumetricamente no meio, devido ao atrito molecular (RODRIGUEZ-JASSO et al., 2011). Esse mecanismo se origina da rotação dipolar de solventes polares e da migração condutora de íons dissolvidos, aumentando o coeficiente de transferência de massa dos ingredientes-alvo (PANDIT et al., 2015). Assim, o efeito direto das microondas nas moléculas através da ocorrência simultânea de polarização dipolar e condução iônica pode transformar a energia do microondas em térmica, levando a um aquecimento quase imediato da amostra e à extração dos compostos de dentro da matriz do material, em direção à solução (MIRZADEH et al., 2020).

Tal fato, resulta na extração mais eficiente dos compostos intracelulares e em comparação aos métodos convencionais (como maceração e refluxo), destacando-se a redução do uso de solventes orgânicos, menores tempos de extração e aumento dos rendimentos obtidos (CHEMAT; CRAVOTTO, 2013). Ademais, este método pode ser aplicado a uma ampla gama de matrizes vegetais, não sendo reportada a degradação de seus compostos funcionais (TEO et al., 2009).

Vislumbrando avaliar a eficiência do processo de EAM do esteviosídeo e reb A da Stevia rebaudiana Bertoni, Jaitak et al. (2009) compararam-na às técnicas de extração convencional e EAU, usando metanol, etanol e água como solventes simples, bem como em misturas binárias. As extrações foram conduzidas, convencionalmente à $25^{\circ} \mathrm{C}$ por $12 \mathrm{~h}$, por ultrassom à 
$35 \pm 5{ }^{\circ} \mathrm{C}$ por 30 min e microondas, à $80 \mathrm{~W}, 50{ }^{\circ} \mathrm{C}$ por $1 \mathrm{~min}$, onde rendimentos na ordem de 4,20 e 1,98\%, 6,54 e 1,20\% e 8,64 e 2,34\% em esteviosídeo e reb A foram obtidos, respectivamente.

Recentemente, rendimentos apreciáveis também foram relatados quando realizada a extração por meio de processos combinados. Görgüç et al. (2019) avaliou a extração enzimática assistida por microondas dos glicosídeos de esteviol e compostos fenólicos das folhas de Stevia rebaudiana Bertoni por meio da metodologia de superfície de resposta (RSM), considerando os efeitos da concentração enzimática da Viscozyme L (1,2-60 FBG unidade $\mathrm{g}^{-1}$ ), temperatura $\left(25-60{ }^{\circ} \mathrm{C}\right)$ e tempo (1-20 min) na otimização das condições e do processo. Os rendimentos máximos em esteviosídeo, reb A e compostos fenólicos totais obtidos foram determinados como 62,5, 25,6 e 20,7 mg/g-1 ${ }^{-1}$, respectivamente, em condições ótimas estimadas em 10,9 unidades $\mathrm{FBG} \mathrm{g} \mathrm{g}^{-1}, 53{ }^{\circ} \mathrm{C}$ e 16 min de processamento.

Corroborando com as informações contidas na maioria dos trabalhos da literatura, o método de extração por microondas apresenta como vantagem uma taxa de extração mais alta, o que promove uma eficiência maior do processo em menores tempos de extração, em comparação com outras tecnologias como EAU, extração por líquido pressurizado (ELP) e extração por fluido supercrítico (EFS), principalmente devido ao aquecimento rápido que ocorre no sistema (ZENG et al., 2012). Entretanto, apesar de haverem relatos sobre a possível industrialização dos processos envolvendo esta tecnologia, principalmente atrelando-a a propriedades antimicrobianas efetivas para pasteurização e esterilização, esta técnica enfrenta a limitação do alto custo do equipamento industrial e da energia necessária para aquecimento necessário pelo microondas (BOYE; ARCAND, 2012).

\section{Extração por eletrotecnologias}

Nos últimos anos, o uso de tecnologias elétricas pulsadas, especialmente campos elétricos pulsados (CEP) e descargas elétricas de alta voltagem (DEAV), têm se mostrado uma ferramenta potencial para recuperação de compostos de matrizes vegetais, principalmente no âmbito de extrações "verdes" e sustentáveis (PARNIAKOV et al., 2014; BARBA et al., 2015a). $\mathrm{O}$ CEP consiste em um tratamento elétrico por um curto período de tempo (de nano à milissegundos), com amplitude de pulso de 100-300 V/cm a 20-80 kV/cm, onde a membrana biológica da matriz vegetal é perfurada eletricamente e perde sua semipermeabilidade temporária ou permanentemente (DENG et al., 2014; BARBA et al., 2015b). As DEAV, em contrapartida, são baseadas em reações químicas e processos físicos. Quando a DEAV é produzida diretamente sobre a mistura da extração, ela injeta energia através de um canal de 
plasma, causando uma descarga elétrica de alta tensão (>40 kV; >10 kA) entre dois eletrodos submersos (BOUSSETTA et al., 2013; BOUSSETTA; VOROBIEV, 2014; BARBA et al., $2015 b$ ), que destroem a parede celular da matriz vegetal.

Os efeitos deste tipo de tecnologia foram avaliados por Duval et al. (2011) na extração dos glicosídeos de esteviol das folhas de Stevia rebaudiana. Os autores constataram que o prétratamento por meio dos CEP $(20 \mathrm{kV} / \mathrm{cm}$ por $0,5-2 \mathrm{~ms})$ antes da extração convencional com água $\left(20^{\circ} \mathrm{C}\right)$, melhorou a cinética, bem como o rendimento da extração, sendo a maximização do processo obtida sob redução significativa no tempo (9 vezes menos) em comparação às amostras não tratadas. O mesmo foi observado quando avaliada a influência destes prétratamentos sob temperaturas moderada $\left(60^{\circ} \mathrm{C}\right)$ e alta $\left(80^{\circ} \mathrm{C}\right)(\mathrm{NEGM}$ et al., 2013).

Ao comparar os efeitos dos CEP e da DEAV na recuperação de polifenóis dos extratos aquosos de stevia, Barba et al. (2015a) verificaram que ambos os tratamentos em comparação com o controle aumentaram o teor de polifenóis totais proporcionalmente à descarga de energia aplicada ao meio $(0,24,47,71,94,118$ e $141 \mathrm{~kJ} / \mathrm{kg})$, em tempos de tratamento equivalentes $\left(120 \mathrm{~min} / 20^{\circ} \mathrm{C}\right)$. Entretanto, a DEAV foi mais eficaz para recuperar os polifenóis $(14,22-29,14$ mg/g EAG), em comparação com os CEP (10,28-20,85 mg/g EAG).

Ao avaliarem e compararem a influência dos pré-tratamentos por CEP e DEAV e mistura binária de água e etanol (0-50\%) na extração dos glicosídeos de esteviol e compostos antioxidantes das folhas de Stevia rebaudiana $\left(1 \mathrm{~h} / 20{ }^{\circ} \mathrm{C} / 100 \mathrm{rpm}\right)$, Carbonell-Capella et al. (2016) constataram capacidade antioxidante total superior nos extratos obtidos por meio da DEAV (87,2 mmol ET/g) na ausência de etanol (0\%) em relação ao extrato obtido por meio do CEP (66,9 mmol ET/g). Em relação à recuperação de esteviosídeos e reb A, os extratos obtidos por meio da extração hidroalcoólica (50\%) submetidas ao CEP, apresentaram conteúdo superior $(\sim 45,0 \mathrm{e} \sim 23,0 \mathrm{mg} / \mathrm{g})$ que as amostras tratadas com DEAV $(\sim 32,0 \mathrm{e} \sim 17,0 \%)$, respectivamente, indicando neste caso, influência direta do solvente na eficiência da extração dos adoçantes.

Embora os resultados da aplicação deste tipo de tecnologia sejam promissores, a maior limitação envolta à esse processo para uma possível aplicação industrial, está relacionada a sua viabilidade, uma vez que demanda alto custo para implantação e operação (TÖPFL, 2006) e, embora a DEAV seja mais eficiente para a desintegração celular que os CEP, há relatos da produção de radicais livres à mistura, o que reduz a atividade antioxidante total do extrato (KOUBAA et al., 2015). Apesar destes inconvenientes, vale ressaltar a importância de que novos passos sejam dados neste segmento, visando assim uma abordagem mais ampla do tema. 
Em meio pressurizado se pode trabalhar com o fluido em estado subcrítico, crítico ou supercrítico. O estado subcrítico ocorre quando a pressão ou a temperatura está abaixo da sua temperatura crítica (Tc) ou pressão crítica (Pc) e o estado supercrítico, quando a temperatura e a pressão dos fluidos ficam acima destes (SANTOS et al., 2015). A Tc é definida como a mais alta temperatura na qual um gás pode ser convertido em líquido, devido a um aumento de pressão. A Pc consiste na mais alta pressão na qual um líquido pode ser convertido em um gás, devido a um aumento de temperatura. Essas propriedades caracterizam o ponto crítico (PC) (BRUNNER, 1994; TAYLOR, 1996).

Acima desse ponto, existe a região supercrítica, na qual o composto apresenta-se como um fluido de uma única fase, não condensável, não havendo distinções entre a fase líquida e a fase gasosa e exibindo algumas propriedades físico-químicas típicas de gases e outras típicas de líquidos (CAVALCANTI, 2013). Em geral, na extração por fluido supercrítico (EFS), as propriedades físicas na região crítica aumentam os processos de transferência de massa e calor, onde, à medida que a pressão aumenta, a compressibilidade da fase gasosa diminui e a densidade aumenta. De tal modo, à medida que a temperatura aumenta, a viscosidade e a densidade da fase líquida diminuem (MONTOYA et al., 2019).

A extração com líquido pressurizado (ELP) consiste na utilização de temperaturas acima do ponto de ebulição dos solventes, os quais são pressurizados de forma a serem mantidos no estado líquido (KOUBAA et al., 2015). Os altos valores de temperatura aumentam a solubilidade, taxa de difusão e transferência de massa, enquanto os valores de viscosidade e tensão superficial do solvente são menores do que aqueles à temperatura ambiente (KOUBAA et al., 2015).

Além dos solventes comumente utilizados neste processo, como etanol e metanol, a água tem sido cada vez mais utilizada na extração, designando ao processo a termologia de extração com água quente pressurizada (EAQP) ou extração com água subcrítica (EASB). A água pressurizada apresenta variação significativa de sua polaridade com a temperatura, o que permite alcançar a extração seletiva de compostos orgânicos polares, moderadamente polares e não polares, somente por meio da alteração dos parâmetros de extração, como temperatura e pressão (LIANG; FAN, 2013).

Visando otimizar a extração dos glicosídeos das folhas de Stevia rebaudiana usando extração com fluido supercrítico (SFE), Erkucuk et al. (2009), por meio de um delineamento experimental, avaliaram o efeito da pressão (150-350 bar), temperatura $\left(40-80{ }^{\circ} \mathrm{C}\right) \mathrm{e}$ concentração da mistura etanol-água (70:30) como co-solvente (0-20\%) pela vazão de $\mathrm{CO}_{2}$ de 
$15 \mathrm{~g} / \mathrm{min}^{-1}$ por $60 \mathrm{~min}$. As condições ótimas de extração foram obtidas sob $211 \mathrm{bar}, 80{ }^{\circ} \mathrm{C}$ e 17,4\% de co-solvente, onde 36,66 mg/g de esteviosídeo e 17,79 mg/g de reb A foram extraídos, compondo valor total em glicosídeos superior à extração etanólica convencional $(48,50 \mathrm{mg} / \mathrm{g})$.

De igual modo, aplicando um delineamento experimental, Yildiz-Ozturk et al. (2014) avaliaram os efeitos da temperatura $\left(100-150{ }^{\circ} \mathrm{C}\right)$, tempo $(30-60 \mathrm{~min})$ e vazão $(2-6 \mathrm{~mL} / \mathrm{min})$, sob pressão de 230 bar à razão sólido/líquido de 1:10 (m:v) frente a extração com água subcrítica da Stevia. A maximização do processo extraiu 38,67 mg/g de esteviosídeo e 35,68 $\mathrm{mg} / \mathrm{g}$ de reb $\mathrm{A}$, à $125^{\circ} \mathrm{C}$, vazão de $4 \mathrm{~mL} / \mathrm{min}$, transcorridos $45 \mathrm{~min}$ de extração. Este extrato apresentou conteúdo total de fenólicos, flavonóides e atividade antioxidante de 48,63 mg de EAG/g de extrato, $29,81 \mathrm{mg}$ de quercetina/g de extrato e 92,50\%, respectivamente. Em termos de clorofila total, conteúdo de carotenóides e fibra alimentar, valores na ordem de 31,91 mg/100 $\mathrm{g}, 5,71 \mathrm{mg} / 100 \mathrm{~g} \mathrm{e} 4,98 \%$ foram encontrados na fase refinada, respectivamente.

Embora existam algumas aplicações industriais para EFS (ROMBAUT et al., 2014), seu uso na indústria alimentícia permanece limitado, devido alto custo do equipamento, instalação (GALANAKIS et al., 2015) e processamento, principalmente quando relacionado com o elevado consumo de solvente (KOUBAA et al., 2015). Para a stevia, a melhora do processo, vislumbrando melhores condições e rendimentos superiores à extração convencional, configuram o principal desafio na aplicação em escala industrial desta tecnologia (ERKUCUK et al., 2009).

Em suma, ao realizar a extração a partir de fluido subcrítico, crítico ou supercrítico, são eminentes as vantagens constatadas em relação a outros métodos, como a alta e rápida taxa de extração, contínua e fácil operação e obtenção dos produtos finais, por dispensar a etapa de filtragem, lavagem e purificação (BUBALO et al., 2018; GALLEGO et al., 2019), além do solvente poder ser facilmente removido do soluto através da redução da pressão e/ou ajuste da temperatura (VASCONCELLOS, 2007), o que promove a estes processos, destaques econômico e ambiental. Porém, salientam-se desafios e a necessidade de investigações que abordem este tema no uso destas tecnologias para a Stevia rebaudiana Bertoni, uma vez que ainda são escassos os trabalhos que contemplam este tema para esta matriz vegetal.

\section{CONCLUSÕES}

A partir da revisão da literatura foi possível observar o aumento da importância no estudo de formas de obtenção dos compostos de interesse da stevia, uma vez que se tem verificado um grande avanço científico envolvendo os estudos de plantas que apresentem 
compostos com propriedades terapêuticas na última década. O processo empregado na obtenção

dos glicosídeos de esteviol vem sendo comumente realizado através dos métodos convencionais de prensagem, maceração, infusão e percolação, sendo possível verificar que estes métodos apresentam alguns inconvenientes, como o longo tempo de extração, menor rendimento e a necessidade de grandes quantidades de solvente, se comparados à métodos não convencionais de extração, como o ultrassom, micro-ondas, eletrotecnologias e em meio pressurizado.

Dentre as vantagens relacionadas a estes métodos, pode-se citar a forma de obtenção e recuperação eficiente, que não cause tantas perdas em seus processos, como a degradação de compostos que levem à consequências negativas na qualidade do produto, possibilidade de eliminação das etapas de filtragem e lavagem, além da alta e rápida taxa de extração, produção limpa, operação fácil e contínua e a possibilidade de emprego de diversos solventes e água, contemplando assim, à estes processos, a caracterização efetiva de "extração verde".

\section{REFERÊNCIAS}

BARBA, F. J.; GRIMI, N.; NEGM, M.; QUILEZ F.; VOROBIEV, E. Green recovery technology of sweeteners from Stevia rebaudiana Bertoni leaves. Leaf Sweeteners: Resources, Processing and Health Effects, v. 01, p. 41-55, 2014.

BARBA, F. J.; GRIMI, N.; VOROBIEV, E. Evaluating the potential of cell disruption technologies for green selective extraction of antioxidant compounds from Stevia rebaudiana Bertoni leaves. Journal of Food Engineering, v. 149, p. 222-228, 2015 a.

BARBA, F. J.; GRIMI, N.; VOROBIEV, E. New approaches for the use of non-conventional cell disruption technologies to extract potential food additives and nutraceuticals from microalgae. Food Engineering Reviews, v. 7, n. 1, p. 45-62, 2015 b.

BERK, Z. Food Process Engineering and Technology. Food Science and Technology, International Series. Elsevier Inc, Second Edition, USA, 2013, p. 287-310.

BUBALO, C. M.; VIDOVIĆ, S.; REDOVNIKOVIĆ, I. R.; JOKIĆ, S. New perspective in extraction of plant biologically active compounds by green solvents. Food and Bioproducts Processing. Food and Bioproducts Processing, v. 109, p. 52-73, 2018.

BOUSSETTA, N.; LESAINT, O.; VOROBIEV, E. A study of mechanisms involved during the extraction of polyphenols from grape seeds by pulsed electrical discharges. Innovative Food Science Emerging Technology, v. 19, p. 124-132, 2013.

BOUSSETTA, N.; VOROBIEV, E. Extraction of valuable biocompounds assisted by high voltage electrical discharges: A review. Comptes Rendus Chimie, v. 17, n. 3, p. 197-203, 2014.

BOYE, J. I.; ARCAND, Y. Green Technologies in Food Production and Processing; 
Springer Science + Business Media, LLC: New York, 2012.

BRAHMACHARI, G.; MANDAL, L. C.; ROY, R.; MONDAL, S.; BRAHMACHARI, A. K. Stevioside and related compounds - molecules of pharmaceutical promise: a critical overview. Arch Pharm (Weinheim), v. 344, p. 5-19, 2011.

BRUNNER, G. Gas extraction: an introduction to fundamentals of supercritical fluids ant the application to separation processes. 1st. Ed. Darmstadl: Steinkopff, New York: Springer, p. 387, 1994.

CARAKOSTAS, M. C.; CURRY, L. L.; BOILEAU, A. C.; BRUSICK, D. J. Overview: The history, technical function and safety of rebaudioside A, a naturally occurring steviol glycoside, for use in food and beverages. Food and Chemical Toxicology, v. 46, p. 1-10, 2008.

CARBOnEll-CAPEllA, J. M.; BARBA, F. J.; ESTEVE, M. J.; FRÍGOLA, A. High pressure processing of fruit juice mixture sweetened with Stevia rebaudiana Bertoni: Optimal retention of physical and nutritional quality. Innovative Food Science \& Emerging Technologies, v. 18, p. 48-56, 2013.

CARBONELL-CAPELLA, J. M.; ŽLABUR, J. Š.; BRNČIĆ, S. R.; BARBA, F. J.; GRIMI, N.; KOUBAA, M.; BRNČIĆ, M.; VOROBIEV, E. Electrotechnologies, microwaves, and ultrasounds combined with binary mixtures of ethanol and water to extract steviol glycosides and antioxidant compounds from Stevia rebaudiana leaves. Journal of Food Processing and Preservation, v. 9, p. 1159-1168, 2016.

CAVALCANTI, R. N. Extração de antocianinas de resíduo de jabuticaba (Myrciaria cauliflora) utilizando líquido pressurizado e fluido supercrítico: caracterização química, avaliação econômica e modelagem matemática. Tese de doutorado em Engenharia de Alimentos. Universidade Estadual de Campinas (UNICAMP). 2013.

CHATSUDTHIPONG, V.; MUANPRASAT, C. Stevioside and related compounds Therapeutic benefits beyond sweetness. Pharmacology \& Therapeutics, v. 121, p. 41-54, 2009.

CHEMAT, F.; ZIIL-e, -H.; KHAN, M. K. Applications of ultrasound in food technology: Processing, preservation and extraction. Ultrasonics Sonochemistry, v. 18, n. 4, p. 813-835, 2011.

CHEMAT, F.; CRAVOTTO, G. In Microwave-Assisted Extraction for Bioactive Compound; Chemat, F., Cravotto, G., Eds.; Springer: New York, 2013.

CHEMAT, F.; ROMBAUT, N.; SICAIRE, A. G.; MEULLEMIESTRE, A.; FABIANOTIXIER, A. N.; ABERT-VIAN, M. Ultrasound assisted extraction of food and natural products. Mechanisms, techniques, combinations, protocols and applications: A review. Ultrasonics Sonochemistry, v. 34, p. 540-560, 2017.

CRIADO, M. N.; BARBA, F. J.; FRÍGOLA, A.; RODRIGO, D. Effect of Stevia rebaudiana on Oxidative Enzyme Activity and Its Correlation with Antioxidant Capacity and Bioactive Compounds. Food Bioprocess Technology, v. 7, p. 1518-1525, 2014. 
DACOME, A. S.; SILVA, C. C.; COSTA, C. E. M.; FONTANA, J. D.; ADELMANN, J.; COSTA, S. C. Sweet diterpenic glycosides balance of a new cultivar of Stevia rebaudiana (Bert.) Bertoni: Isolation and quantitative distribution by chromatographic, spectroscopic, and eletrophoretic methods. Process Biochemistry, v. 40, p. 3587-3594, 2005.

DENG, Q.; ZINOVIADOU, K. G.; GALANAKIS, C. M.; ORLIEN, V.; GRIMI N.; VOROBIEV, E.; LEBOVKA, N.; BARBA, F. J. The effects of conventional and nonconventional processing on glucosinolates and its derived forms, isothiocyanates: extraction, degradation, and applications. Food Engineering Reviews, v. 7, p. 357-381, 2015.

DUVAL, Q.; GRIMI, N.; VOROBIEV, E. Intensification d'un procédé de production du stevioside à partir des feuilles de stevia. Rapp. PTV, 50, 2011.

ERKUCUK, A.; AKGUN, H.; YESIL-CELIKTAS, O. Supercritical CO2 extraction of glycosides from Stevia rebaudiana leaves: Identification and optimization. The Journal of Supercritical Fluids, v. 51, n. 1, p. 29-35, 2009.

FERRAZZANO, G. F.; CANTILE, T.; ALCIDI, B.; CODA, M.; INGENITO, A.; ZARRELLI, A.; DI FABIO, G.; POLLIO, A. Is Stevia rebaudiana Bertoni a non cariogenic sweetener? A review. Molecules, v. 21, p. 1-12, 2016.

FORMIGONI, M.; MILANI, P. G.; DACOME, A. S.; COSTA, S. C. Effect of enzymatic pretreatment on the extraction yield of Stevia rebaudiana leaves. International Food Research Journal, v. 25, n. 4, p. 1510-1514, 2018a.

FORMIGONI, M.; MILANI, P. G.; AVINCOLA, A.; SANTOS, V. J.; MARCHI, L. B.; DACOME, A. S.; PILAU, E.; COSTA, S. C. Pretreatment with ethanol as an alternative to improve steviol glycosides extraction and purification from a new variety of stevia. Food Chemistry, v. 241, p. 452-459, 2018 b.

FORMIGONI, M.; COSTA, S. C.; ZORZENON, M. R. T.; MILANI, P. G.; RASPE, D. T.; CIOTTA, S.; DACOME, A. S. Conventional extraction techniques. In: Stevio Glycosides: Production, Properties and Applications. Elsevier. 1ed. 2020.

GALANAKIS, C. M.; BARBA, F. J.; PRASAD, K. N. Cost and Safety Issues of Emerging Technologies against Conventional Techniques. Food Waste Recovery Processing Technologies and Industrial Techniques, p. 323-338, 2015.

GASMALLA, M. A. A.; YANG, R.; MUSA, A.; HUA, X.; YE, F. Influence of sonication process parameters to the state of liquid concentration of extracted rebaudioside $A$ from Stevia (Stevia rebaudiana Bertoni) leaves. Arabian Journal of Chemistry, v. 10, p. 726-731, 2017.

GÖRGÜÇ, A.; GENÇDAĞ, E.; YILMAZ, F. M. Optimization of microwave assisted enzymatic extraction of steviol glycosides and phenolic compounds from stevia leaf. Acta Periodica Technologica, v. 50, p. 69-76, 2019.

HENG, M. Y.; TAN, S. N.; YONG, J. W. H.; ONG, E. S. Emerging green technologies for the chemical standardization of botanicals and herbal preparations. Trends in Analytical Chemistry, v. 50, p. 1-10, 2013. 
JAITAK, V.; BANDNA, B. S.; KAUL, V. K. An efficient microwave-assisted extraction process of stevioside and rebaudioside-A from Stevia rebaudiana (Bertoni). Phytochemical Analysis, v. 20, p. 240-245, 2009.

JEPPESEN, P. B.; GREGERSEN, S.; POULSEN, C. R.; HERMANSEN, K. Stevioside acts directly on pancreatic $\beta$-cells to secrete insulin: Actions independent of cyclic adenosine monophosphate and adenosine triphosphate-sensitive K+ channel activity. Metabolism, v. 49, p. 208-214, 2000.

KHODDAMI, A.; WILKES, M. A.; ROBERTS, T. H. Techniques for analysis of plant phenolic compounds. Molecules, v. 18, n. 2, p. 2328-2375, 2013.

KOUBAA, M.; ROSELLÓ-SOTO, E.; ŠIC ŽLABUR, J.; JAMBRAK, A. R.; BRNČIĆ, M.; GRIMI, N.; BOUSSETTA, N.; BARBA, F. J. Current and new insights in the sustainable and green recovery of nutritionally valuable compounds from Stevia rebaudiana Bertoni. Journal of Agricultural Food and Chemistry, v. 63, n. 31, p. 6835-6846, 2015.

KOVACEVIC, D. B.; BARBA, F. J.; GRANATO, D.; GALANAKIS, C. M.; HERCEG, Z.; DRAGOVIC-UZELAC, V.; PUTNIK, P. Pressurized Hot Water Extraction (PHWE) for the Green Recovery of Bioactive Compounds and Steviol Glycosides from Stevia rebaudiana Bertoni Leaves. Food Chemistry, v. 254, p. 150-157, 2018.

KOVYLYAEVA, G. I.; BAKALEINIK, G. A.; STROBYKINA, I. YU.; GUBSKAYA, V. I.; SHARIPOVA, R. R.; AL'FONSOV, V. A.; KATAEV, V. E.; TOLSTIKOV, A. G. Glycosides from Stevia rebaudiana. Chemistry of Natural Compounds, v. 43, n. 1, p. 81-85, 2007.

LIANG, X.; FAN, Q. Application of sub-critical water extraction in pharmaceutical industry. Journal of Materials Science and Chemical Engineering, v. 1, p. 1-6, 2013.

LIU, J., LI, J.-W., TANG, J. Ultrasonically assisted extraction of total carbohydrates from Stevia rebaudiana Bertoni and identification of extracts. Food and Bioproducts Processing, v. 88, p. 215-221, 2010.

LOVRIC, V.; PUTNIK, P.; KOVAČEVIĆ, D. B.; JUKIĆ, M.; DRAGOVIĆ-UZELAC, V. Effect of Microwave-Assisted Extraction on the Phenolic Compounds and Antioxidant Capacity of Blackthorn Flowers. Food Technology and Biotechnology, v. 55, n. 2, p. 243$250,2017$.

MAHNIC-KALAMIZA, S.; VOROBIEV, E.; MIKLAVČIČ, D. Electroporation in Food Processing and Biorefinery. The Journal of Membrane Biology, v. 247, p. 1279-1304, 2014.

MCDONNELL, C.; TIWARI, B. K. Chapter Five - Ultrasound: A Clean, Green Extraction Technology for Bioactives and Contaminants. Comprehensive Analytical Chemistry, n. 6, p. 11-129, 2017.

MILANI, P. G.; FORMIGONI, M.; LIMA, Y. C.; PIOVAN, S.; PEIXOTO, G. M. L.; CAMPARSI, D. M.; DA SILVA RODRIGUES, W. N.; DA SILVA, J. Q. P.; DA SILVA AVINCOLA, A.; PILAU, E. J.; DA COSTA, C. E. M.; DA COSTA, S. C. Fortification of the whey protein isolate antioxidant and antidiabetic activity with fraction rich in phenolic compounds obtained from Stevia rebaudiana (Bert.). Bertoni leaves. Journal of Food Science and Technology, v. 54, p. 2020-2029, 2017. 
MILIĆ, P. S.; RAJKOVIĆ, K. M.; STAMENKOVIĆ, O. S.; VELJKOVIĆ, V. B. Kinetic modeling and optimization of maceration and ultrasound-extraction of resinoid from the aerial parts of white lady's bedstraw (Gallium mollugo L.). Ultrasonics Sonochemistry, v. 20, p. $525-534,2013$.

MIRZADEH, M.; ARIANEJAD, M. R.; KHEDMAT, L. Antioxidant, antiradical, and antimicrobial activities of polysaccharides obtained by microwave-assisted extraction method: a review. Carbohydrate Polymers, v. 229, n. 1, p. 1-39, 2020.

MONTOYA, M. F., SÁNCHEZ, F. A.; PEREDA, S. Equilibrium in Pressurized Systems (Sub and Supercritical). In: PEREIRA, C. G. Thermodynamics of Phase Equilibria in Food Engineering, Cambridge: Academic Press. p. 385-418, 2019.

NEGM, M.; GRIMI, N.; VOROBIEV, E. Pulsed Electric Field Pretreatment Assisted the Green Solvent Extraction of Sweet Glycosides from Stevia rebaudiana. In International Congress on Green Extraction of Natural Products; GENP Avignon: France, 2013.

PANDIT, S. G.; VIJAYANAND, P.; KULKARNI, S. G. Pectic principles of mango peel from mango processing waste as influenced by microwave energy. LWT - Food Science and Technology, v. 64, n. 2, p. 1010-1014, 2015.

PARNIAKOV, O.; BARBA, F. J.; GRIMI, N.; LEBOVKA, N.; VOROBIEV, E. Impact of pulsed electric fields and high voltage electrical discharges on extraction of high-added value compounds from papaya peels. Food Research International, v. 65 (Part C), p. 337-343, 2014.

PASSOS, C. P.; SILVA, R. M.; SILVA, F. S.; COIMBRA, M. A.; SILVA, C. M. Supercritical fluid extraction of grape seed (Vitis vinifera $\mathrm{L}$.) oil. Effect of the operating conditions upon oil composition and antioxidant capacity. Chemical Engineering Journal, v. 160, n. 2, p. 634$640,2010$.

POL, J.; OSTRA, E. V.; KARASEK, P.; ROTH, M.; BENESOVA, K.; KOTLARIKOVA, P. Comparison of two different solvents employed for pressurized fluid extraction of stevioside from Stevia rebaudiana: methanol versus water. Analytical and Bioanalytical Chemistry, v. 388, p. 1847-1857, 2007.

PRADO, J.M.; VEGGI, P.C.; MEIRELES, M.A.A. Scale-Up Issues and Cost of Manufacturing Bioactive Compounds by Supercritical Fluid Extraction and Ultrasound Assisted Extraction. In: Babosa-Cánovas G. et al. (Eds) Global Food Security and Wellness. Springer, New York, p. 377-433, 2017.

PINGRET, D.; FABIANO-TIXIER, A.-S.; BOURVELLEC, C. L.; RENARD, C. M. G. C.; CHEMAT, F. Lab and pilot-scale ultrasound-assisted water extraction of polyphenols from apple pomace. Journal of Food Engineering, v. 111, n. 1, p. 73-81, 2012a.

PINGRET, D.; DURAND, G.; FABIANO-TIXIER, A.-S.; ROCKENBAUER, A.; GINIES, C.; CHEMAT, F. Degradation of edible oil during food processing by ultrasound: electron paramagnetic resonance, physicochemical, and sensory appreciation. Journal of Agricultural and Food Chemistry, v. 60, n. 31, p. 7761-7768, 2012 b. 
PURI, M.; SHARMA, D. Antibacterial activity of stevioside towards food-borne pathogenic bacteria. Engineering in Life Sciences, v. 11, n. 3, p. 326-329, 2011.

PURI, M.; SHARMA D.; TIWARI, A. K. Downstream processing of stevioside and its potential applications. Biotechnology Advances, v. 29, p. 781-791, 2011.

PURI, M.; SHARMA, D.; BARROW, C. J.; TIWARY, A. K. Optimisation of novel method for the extraction of steviosides from Stevia rebaudiana leaves. Food Chemistry, v. 132, p. 1113-1120, 2012.

PUTNIK, P.; BURSAĆ KOVAČEVIĆ, D.; DRAGOVIĆ-UZELAC, V. Optimizing acidity and extraction time for polyphenolic recovery and antioxidant capacity in grape pomace skin extracts with response surface methodology approach. Journal of Food and Process Preservation, v. 40, n. 6, p. 1256-1263, 2016.

RODRIGUEZ-JASSO, R. M.; MUSSATTO, S. I.; PASTRANA, L.; AGUILAR, C. N.; TEIXEIRA, J. A. Microwave-assisted extraction of sulfated polysaccharides (fucoidan) from brown seaweed. Carbohydrate Polymers, v. 86, n. 3, p. 1137-1144, 2011.

ROMBAUT, N.; TIXIER, A.-S.; BILY, A.; CHEMAT, F. Green extraction processes of natural products as tools for biorefinery. Biofuels, Bioproducts and Biorefining, v. 8, n. 4, p. 530-544, 2014.

SANTOS, K. A.; BARICCATTI, R. A.; CARDOZO-FILHO, L.; SCHNEIDER, R.; PALÚ, F.; SILVA, C.; SILVA, E. A. Extraction of crambe seed oil using subcritical propane: Kinetics, characterization and modeling. Journal of Supercritical Fluids, v. 104, p. 54-61, 2015.

SHARMA, D.; PURI, M.; TIWARI, A. K.; SINGH, N.; JAGGI, A. S. Antiamnesic effect of stevioside in scopolamine-treated rats. Indian Journal of Pharmacology, v. 42, p. 164-167, 2010.

TAKAHASHI, K.; MATSUDA, M.; OHASHI, K.; TANIGUCHI, K.; NAKAGOMI, O.; ABE, Y.; MORI, S.; SATO, N.; OKUTANI, K.; SHIGETA, S. A. Analysis of anti-rotavirus activity of extract from Stevia rebaudiana. Antiviral Research, v. 49, n. 1, p. 15-24, 2001.

TAYLOR, L. T. Supercritical fluid extraction. New York: John Wiley \& Sons Inc., p. 181, 1996.

TEO, C. C.; TAN, S. N.; YONG, J. W. H.; HEW, C. S.; ONG, E. S. Validation of greensolvent extraction combined with chromatographic chemical fingerprint to evaluate quality of Stevia rebaudiana Bertoni. Journal of Separation Science, v. 32, n. 4, p. 613-622, 2009.

TÖPFL, S. Pulsed Electric Fields (PEF) for Permeabilization of Cell Membranes in Food and Bioprocessing Applications. Process and Equipment Design and Cost Analysis; Berlin University of Technology: Berlin, 2006.

TZIA, C.; IADAKIS, G.; AGUILERA, J. M. Extraction Optimization in Food Engineering. Marcel Dekker, Inc. USA, 2003, p. 33-34. 
VASCONCELLOS, C. M. C. Extração supercrítica dos óleos voláteis de Achyrocline satureioides (Macela) e Vetiveria zizanioides (Vetiver): determinação da cinética de extração e estimativa de custos de manufatura. Dissertação de Mestrado em Engenharia de Alimentos. Faculdade de Engenharia de Alimentos, Universidade Estadual de Campinas, Campinas, p. 114, 2007.

VINATORU, M.; MASON, T. J.; CALINESCU, I. Ultrasonically Assisted Extraction (UAE) and Microwave Assisted Extraction (MAE) of Functional Compounds from Plant Materials. Trends in Analytical Chemistry, v. 97, p. 159-178, 2017.

VIROT, M.; TOMAO, V.; LE BOURVELLEC, C.; RENARD, C. M. C. G.; CHEMAT, F. Towards the industrial production of antioxidants from food processing by-products with ultrasound-assisted extraction. Ultrasonics Sonochemistry, v. 17, n. 6, p. 1066-1074, 2010.

WILLIAMS, L. D.; BURDOCK, G. A. Genotoxicity studies on a high-purity rebaudioside A preparation. Food and Chemical Toxicology, v. 47, p. 1831-1836, 2009.

WÖLWER-RIECK U. The Leaves of Stevia rebaudiana (Bertoni), Their Constituents and the Analyses Thereof: A Review. Journal of Agricultural and Food Chemistry, v. 60, p. 886$895,2012$.

YILDIZ-OZTURK, E.; TAG, O.; YESIL-CELIKTAS, O. Subcritical water extraction of steviol glycosides from Stevia rebaudiana leaves and characterization of the raffinate phase. Supercritical Fluids, v. 95, p. 422-430, 2014.

YU, H.; YANG, G.; SATO, M.; YAMAGUCHI, T.; NAKANO, T.; XI, Y. Antioxidant activities of aqueous extract from Stevia rebaudiana stem waste to inhibit fish oil oxidation and identification of its phenolic compounds. Food Chemistry, v. 232, p. 379-386, 2017.

ZENG, W. C.; ZHANG, Z.; GAO, H.; JIA, L. R.; CHEN, W. Y. Characterization of antioxidant polysaccharides from Auricularia auricular using microwave-assisted extraction. Carbohydrate Polymers, v. 89, n. 2, p. 694-700, 2012.

ŽLABUR, J. Š.; VOĆA1, S.; DOBRIČEVIĆ, N.; BRNČIĆ, M.; DUJMIĆ, F.; BRNČIĆ, S. R. Optimization of ultrasound assisted extraction of functional ingredients from Stevia rebaudiana Bertoni leaves. International Agrophysics, v. 29, p. 231-237, 2015. 